\title{
EFEITOS DO MEIO SOBRE A QUALIDADE DA MATURAÇÃO NUCLEAR IN VITRO DE OÓCITOS BOVINOS OBTIDOS DE ABATEDOURO
}

Elcio Ricardo José de Sousa Vicente ${ }^{1}$, Aline Nochi Berto ${ }^{2}$, Gabriela Negri ${ }^{2}$, Daniel Briguenti Massuia $^{2}$, Aline de Oliveira Santos ${ }^{3}$, Kleber Luciano Ancioto ${ }^{4}$, Avelino Albas ${ }^{5}$, Caliê Castilho ${ }^{2}$, Sheila Merlo Garcia ${ }^{1}$.

${ }^{1}$ Universidade do Oeste Paulista - UNOESTE, Curso de Zootecnia, Presidente Prudente - SP. ${ }^{2}$ Curso de Medicina Veterinária, Presidente Prudente - SP. ${ }^{3}$ Bolsista FAPESP - APTA/SAA-SP. ${ }^{4}$ Gerente de produção da Gene Up. ${ }^{5}$ Pesquisador da APTA/SAA-SP. E-mail: elcio.ricardo@hotmail.com

\section{RESUMO}

O objetivo da pesquisa que origina este trabalho foi avaliar a influência da adição de antioxidantes ao meio de maturação sobre a maturação nuclear in vitro de oócitos bovinos obtidos de abatedouro. Para sua execução foram utilizados somente ovários de fêmeas bovinas zebuínas. Os folículos foram aspirados e os oócitos recuperados, selecionados e classificados. Utilizaram-se três meios (TCM 199 com Antioxidant Supplement [sigma A-1345], TCM 199 suplementado com ácido ascórbico e TCM 199 suplementado com glutamina); para avaliar a maturação nuclear. Não foi possível verificar a ação protetora ou anti-oxidativa dos tratamentos, pois não houve diferença significativa dos diferentes estádios de maturação. Sugere-se dar continuidade aos experimentos, com objetivo de aumentar o $\mathrm{n}$ Total, para que se possa verificar novamente a existência de possíveis diferenças entre os meios propostos.

Palavras-chave: Maturação in vitro. Bos indicus. Antioxidante. Ácido ascórbico. Glutamina.

\section{EFFECTS OF ENVIRONMENT ON THE QUALITY OF NUCLEAR MATURATION IN VITRO BOVINE OOCYTE OBTAINED SLAUGHTERHOUSE}

\begin{abstract}
The objective of this project was to evaluate the influence of maturation medium on in vitro bovine oocyte nuclear maturation slaughterhouse. For the implementation of this project only ovaries of zebu cows were used. Follicles were aspirated, oocytes retrieved, selected and sorted. The oocytes were matured with three maturation media (TCM 199 with Antioxidant Supplement (Sigma A-1345), TCM 199 supplemented with ascorbic acid and TCM 199 supplemented with glutamine); and used to assess nuclear maturation. In this work, it was not possible to verify the protective or anti-oxidative action of ascorbic acid, or glutamine, as there was no significant influence of culture media on the different stages of maturation. It is suggested to continue the experiments, aiming to increase on Total, so you can check back for possible differences between the means proposed.
\end{abstract}

Keywords: In vitro maturation. Bos indicus. Antioxidant. Ascorbic acid. Glutamine.

\section{INTRODUÇÃO}

A média atual do desenvolvimento in vitro permanece em torno de $30 \%$ do total de oócitos maturados. Para 8 a 10 oócitos colhidos pela aspiração folicular guiada por ultrassonografia (Ovum 
Pick-up, OPU), há uma produção média de dois embriões transferidos em bovinos de raças europeias (GALLI et al., 2000). Em animais da raça Nelore (Bos indicus), Dayan et al. (2000), observaram grande variação na taxa de blastocisto entre as doadoras, com média de $42 \%$ de produção in vitro de embriões (PIVE).

A suplementação do meio de maturação in vitro com fatores de crescimento presentes no ambiente folicular vem sendo utilizada no intuito de tornar o ambiente mais parecido com o que ocorre fisiologicamente.

O conhecimento de fatores externos que influenciam o desenvolvimento e criação de um sistema de produção in vitro sob condições controláveis, pode ajudar a melhorar as taxas de desenvolvimento embrionário in vitro e os resultados de gestação. Esses fatores são fundamentais para indicar alterações necessárias visando tornar o sistema in vitro mais semelhante possível do in vivo e, com isso, melhorar a qualidade dos meios de cultura utilizados na produção in vitro de embriões e aumentar a disponibilidade de oócitos de melhor qualidade para serem utilizados na PIVE.

Os oócitos e embriões parecem estar protegidos do estresse oxidativo pela presença de antioxidantes dos fluidos folicular e do oviduto (ANDRADE et al., 2010). Porém, quando os oócitos são tirados de seu ambiente natural, para a participação na PIVE, eles perdem a sua defesa natural, e, portanto, podem ser tomados cuidados especiais como a adição de antioxidantes ao meio de cultivo, para evitar a ocorrência de estresse oxidativo (WANG et al., 2002).

Os aminoácidos L-glutamina (via glutamato, que é um precursor direto da glutationa), cisteína e glicina são precursores na síntese do tripeptídeo glutationa, L- $\nu$-glutamil-cisteinilglicina, que está presente nas células nas formas reduzida (GSH) e oxidada (GSSG). A molécula de GSH é conhecida por ter múltiplas funções fisiológicas incluindo a defesa antioxidante, regulação da proliferação celular, entre outras (SAND, 2005).

O ácido ascórbico (vitamina C) ou ascorbato é uma vitamina hidrossolúvel com ação antioxidante. Ele é predominantemente um antioxidante solúvel presente no ovário e pode ser encontrado no oócito, nas células da granulosa, da teca interna e luteínicas (THOMAS et al., 2001). O ascorbato atua juntamente com a glutationa para proteger a célula dos danos oxidativos (NORDBERG e ARNÉR, 2001).

Assim, a pesquisa teve como principal objetivo, avaliar a maturação nuclear in vitro de oócitos bovinos, comparando três meios distintos: TCM 199 com Antioxidant Supplement (sigma A-1345); TCM 199 suplementado com ácido ascórbico; e TCM 199 suplementado com glutamina. 


\section{METODOLOGIA}

O experimento foi desenvolvido no laboratório de PIVE do mestrado em Ciência Animal da Universidade do Oeste Paulista - UNOESTE. Foram utilizados ovários, de fêmeas bovinas da raça Nelore e aneloradas, obtidos no abatedouro Bon-Mart Frigorífico Ltda.

Os ovários coletados foram colocados em solução fisiológica, imediatamente enviados ao laboratório de PIVE onde foram lavados com solução salina, e mantidos em banho-maria à temperatura de $37^{\circ} \mathrm{C}$ até a aspiração. Os complexos cumulus-oócito (COC's) foram aspirados de folículos entre 2 e $8 \mathrm{~mm}$ de diâmetro e com boa coloração do fluido folicular. A aspiração foi feita com seringas de $10 \mathrm{ml}$ de volume acopladas a agulhas de $25 \times 8$. O fluido folicular foi depositado em tubos cônico de $15 \mathrm{ml}$ mantido em repouso por aproximadamente 10 minutos em banhomaria a $35^{\circ} \mathrm{C}$, para que houvesse a decantação dos $\mathrm{COC}^{\prime}$ 's e células da granulosa.

Com o auxílio de uma lupa estéreo-microscópica (Forty, American Optical Corporation), os COC's foram selecionados e classificados de acordo com o aspecto morfológico dos complexos cumulus-oócito (COC`s) segundo Lonergan (1992).

Foram cultivados Somente os COC's classificados em grau I e II, que apresentaram citoplasma escuro e homogêneo, podendo ter pequenas irregularidades e com no mínimo três camadas de células do cumulus compactas, uniformes e sem pigmentações. Os COC's selecionados foram lavados em meio TCM-199 HEPES e divididos em quatro grupos.

O grupo 1 não foi submetido à maturação e os grupos 2, 3 e 4, foram colocados para maturar e previamente identificadas conforme com o tratamento. Com o intuito de observar a qualidade dos COC's selecionados avaliou-se o estádio nuclear que esses encontravam-se antes de serem submetidos à maturação.

Os grupos 2, 3 e 4 de COC's foram maturados em placas separadas e identificadas conforme o tratamento. Os meios de maturação utilizados foram: MIV controle (TCM 199, 10\% de soro fetal bovino e Antioxidant Supplement - Sigma A-1345); MIV suplementado com glutamina (TCM 199, 10\% de soro fetal bovino e glutamina [0,3 mg/ $\mu \mathrm{L}]$ ); MIV suplementado com ácido ascórbico (TCM 199, 10\% de soro fetal bovino e ácido ascórbico [0,1 mg/ $\mu \mathrm{L}]$ ). Foram cultivados de 20-25 COC's por gota de $90 \mu \mathrm{L}$ dos meios de maturação, cobertas com óleo mineral, mantidas em estufa com $95 \%$ de umidade, atmosfera gasosa de $5 \%$ de $\mathrm{CO}_{2}$ em ar, a 39 으, por um período de $22 \mathrm{~h}$.

Para a classificação dos estádios de maturação nuclear, após o cultivo, os COC's foram retirados dos respectivos tratamentos, depositados em placas de Petri contendo $0,2 \%$ de 
hialuronidase (Hyalozima ${ }^{\circledR}$, Aspen) e desnudados mecanicamente com pipeta de $100 \mu \mathrm{L}$ até a completa remoção das células do cumulus. Posteriormente, os oócitos foram fixados em solução de $4 \%$ de paraformaldeído por 30 minutos à temperatura ambiente, em placa de quatro poços, permeabilizados por 15 minutos a 38 ㅇ C em solução de $0,1 \%$ de Triton X- 100 e corados com Hoechst $33342(10 \mu \mathrm{g} / \mathrm{mL})$ durante 10 minutos. Após o processo de coloração os oócitos foram lavados em PBS e transferidos para lâminas de vidro, em gotas $10 \mu \mathrm{L}$ de glicerol recobertas com lamínulas.

Os oócitos foram avaliados quanto ao estádio da meiose em microscópio de epifluorescência (Olympus - IX-51), objetiva de 40x, excitação 330-385nm e emissão 420-490nm para o Hoechst, no Laboratório de Pesquisas em Economia e Ciência Animal do Polo Regional de Desenvolvimento Tecnológico dos Agronegócios da Alta Sorocabana (APTA/SAA-SP) em Presidente Prudente-SP.

Os COC's foram classificados em: Imaturos (COC's em vesícula germinativa - VG, quebra da vesícula germinativa - QVG, e metáfase I - MI), Maduros (COC's em metáfase II - MII) e degenerados.

Os dados foram analisados através de testes não-paramétricos, sendo o efeito dos meios de cultivo verificado por meio do teste Qui-quadrado $\left(\chi^{2}\right)$ e as comparações múltiplas com os diferentes meios de cultivo comparados pelo teste exato de Fisher, utilizando Software " $R$ " (R core Team, 2013).

O nível de significância utilizado para rejeitar $\mathrm{H}_{0}$ (hipótese de nulidade) foi de $5 \%$, isto é, para $p<0,05$, considerou-se que ocorreram diferenças significativas entre os tratamentos (meios de cultivo) para um determinado estádio de maturação (VG, QVG, MI, MII e degenerados).

\section{RESULTADOS}

Foram selecionados 427 COC's, (241 grau I e 186 grau II) distribuídos em três experimentos. Na primeira repetição, foram colhidos 21 ovários, selecionando-se 145 COC's; na segunda repetição, obtiveram-se 39 ovários, sendo selecionados 122 COC's; na terceira repetição o número de ovários foi de 40, selecionando-se 160 COC's, totalizando-se, nos três experimentos, 100 ovários.

Do total de COC's selecionados, 142 oócitos foram avaliados quanto ao estádio nuclear, sendo que 55 oócitos eram do grupo de oócitos que não foram maturados; 23 oócitos do grupo que foi maturado no meio controle; 28 oócitos do grupo suplementado com ácido ascórbico; 36 oócitos do grupo suplementado com glutamina. 
Essa diferença entre os oócitos selecionados e avaliados ocorreu devido às perdas durante todo o processo de coloração do núcleo, ou seja, nas pipetagens realizadas para desnudar os oócitos, nas mudanças de etapa do protocolo, nas lavagens, bem como na montagem e visualização das lâminas.

Dos COC's maturados e classificados quanto ao estádio da meiose, foi possível identificar: 32 oócitos classificados como imaturos; 36 oócitos classificados como maduros (em Metáfase II M II); 19 oócitos degenerados (Tabela 01).

Dos oócitos considerados imaturos, observou-se: 11 oócitos em estádio de Vesícula Germinativa (VG); 7 oócitos em Quebra da Vesícula Germinativa (QVG); 14 oócitos em Metáfase I (M I).

Tabela1. Diferenças percentuais nos índices de maturação.

\begin{tabular}{l|c|c|c|c}
\hline Tratamento & $\mathrm{n}$ Total & $\begin{array}{c}\text { Oócitos imaturos } \\
\text { (VG, QGV, M I) }\end{array}$ & $\begin{array}{c}\text { Maduros } \\
\text { (M II) }\end{array}$ & Degenerados \\
\hline Antioxidant Supplement & 23 & $10(43 \%)$ & $7(31 \%)$ & $6(26 \%)$ \\
\hline Ác. Ascórbico & 28 & $8(29 \%)$ & $13(46 \%)$ & $7(25 \%)$ \\
\hline Glutamina & 36 & $14(39 \%)$ & $16(44 \%)$ & $6(17 \%)$ \\
\hline
\end{tabular}

Fonte: Resultados da pesquisa.

Os COC's não maturados tiveram 29\% de oócitos degenerados, sendo um indicativo de má qualidade dos mesmos, também, observou-se que $25 \%$ estavam em VG, $42 \%$ em QVG e $4 \%$ em M I, pois o fato da simples remoção do oócito do ambiente folicular estimula a retomada da meiose (SIRARD et al., 2006). Além disso, não é possível saber em que fase da onda folicular encontravamse os oócitos.

\section{DISCUSSÃO}

Segundo Lonergan et al. (apud Apparício-Ferreira, 2010) em bovídeos, a maturação in vitro (MIV) apresenta taxas próximas de 90\%, mas nesse trabalho, as porcentagens de Maturação, foram respectivamente de $30 \%, 44 \%$ e $46 \%$.

Foram encontrados $26 \%, 17 \%$ e $25 \%$, respectivamente, de oócitos degenerados, isso demonstra uma má qualidade dos mesmos, como já mencionado, levando-se em conta a porcentagem de oócitos degenerados em relação aos oócitos não maturados.

Os oócitos que se encontravam em VG, QVG e MI, que por qualquer motivo foram interrompidos, indicam que houve um início de maturação, pois a maturação nuclear envolve as 
alterações ocorridas durante a passagem da fase de VG para a M II, sendo que o primeiro sinal de reinicio da meiose é caracterizada pela transição do estádio de VG ao de M II.

Para avaliar o efeito dos meios de cultivo sobre os diferentes estádios de maturação foi empregado o teste Qui-Quadrado $\left(\chi^{2}\right)$, para comparação entre a taxa de maturação dos grupos: "sem maturação nuclear", "maturados" e "degenerados". Os grupos "sem maturação nuclear" correspondem à soma dos oócitos que se encontravam em VG, QVG e M I, dos tratamentos analisados e comparados entre si, pelo fato de não terem alcançado a M II, por algum motivo.

Para uma significância de 5\%, o valor do $\chi^{2}$ obtido foi igual a 2,52; portanto, menor que o $\chi^{2}$ crítico $=4 \mathrm{gl}=9,49$. Com base nesse resultado, pode-se afirmar que não houve influência significativa dos meios de cultivo sobre os diferentes estádios de maturação, portanto a hipótese $\mathrm{H}_{0}$ não pode ser rejeitada.

A aquisição de competência por parte dos oócitos é influenciada pelo meio de maturação e seus suplementos (LONERGAN et al, 2003). Existe uma grande variação em relação à composição dos meios de cultura entre os grupos de pesquisa. Embora o soro fetal bovino (SFB) seja comumente adicionado ao meio de MIV, ele contém muitos componentes desconhecidos que podem introduzir uma enorme variabilidade fisiológica ao sistema de MIV, o que impede a padronização necessária para obtenção de uma resposta consistente (CHIAN et al, 2003).

Com relação ao estádio de $\mathrm{M}$ II, dos oócitos avaliados, podemos observar que $31 \%, 46 \%$, 44\% completaram a maturação, respectivamente nos meios suplementados com Antioxidant Supplement (sigma A-1345), ácido ascórbico e glutamina. Além disso, observa-se uma taxa significativa de oócitos degenerados em todos os tratamentos: 25 \% no meio com Antioxidant Supplement e $26 \%$ no meio com ácido ascórbico, porém o meio suplementado com glutamina foi o que obteve número de degeneração (17\%).

A literatura descreve que a presença de antioxidante é essencial para a maturação de oócitos, e necessária para o desenvolvimento embrionário (SILVA, et al., 2011).

Tatemoto et al. (apud SILVA et al, 2011), verificaram que o tratamento com ácido ascórbico, no meio de maturação, protegeu os oócitos suínos do estresse oxidativo e ainda aumentou a formação de pró-núcleos. No entanto, neste trabalho, não foi possível verificar a ação do ácido ascórbico como antioxidante, pelos baixos índices encontrados.

Segundo Ferreira et al. (2008), juntamente com a glutationa, outras moléculas do sistema enzimático antioxidante têm um importante papel atenuante dos efeitos deletérios do estresse oxidativo provocado pelas espécies reativas ao oxigênio (EROs), sendo que já foi demonstrado que a glutationa peroxidase está presente em oócitos bovinos e células do cumulus após a MIV, mas 
não detectou, em sua pesquisa, diferença entre os grupos em relação à produção de EROs. Assim como neste trabalho, não foi possível verificar a ação protetora ou anti-oxidativa da glutamina.

\section{CONCLUSÃO}

Essa pesquisa teve como principal objetivo, avaliar a ação de antioxidantes na maturação nuclear dos oócitos bovinos maturados in vitro, em três meios distintos: TCM 199 Antioxidant Supplement (sigma A-1345); TCM 199 suplementado com ácido ascórbico; e TCM 199 suplementado com glutamina.

A suplementação dos meios de MIV não alcançaram resultados significativos. Portanto, nesse estudo, não foi observado o esperado efeito protetor promovido pela adição de compostos antioxidantes aos meios de maturação oócitária, o qual torna o ambiente mais favorável para aquisição da competência meiótica.

Sugere-se que se deva dar continuidade aos experimentos, com objetivo de aumentar o $\mathrm{n}$ Total, para que se possa verificar novamente a existência de possíveis diferenças entre os meios propostos.

Portanto, mais estudos são necessários para esclarecer qual o melhor meio de maturação nuclear para os oócitos bovinos, e assim, estabelecer um protocolo de maior eficiência, no que diz respeito à competência do oócito e da MIV.

\section{REFERÊNCIAS}

ANDRADE, E. R.; MELO-STERZA, F. A.; SENEDA, M. M.; ALFIERI, A. A. Consequências da produção das espécies reativas de oxigênio na reprodução e principais mecanismos antioxidantes. Rev. Bras. Reprod. Anim., Belo Horizonte, v. 34, n. 2, p. 79-85, abr./jun. 2010. Disponível em: <http://www.cbra.org.br>. Acesso em: 28 mar. 2013.

APPARÍCIO-FERREIRA, M. Maturação nuclear e citoplasmática de oócitos de cadelas colhidos em diferentes fases do ciclo estral e cultivados in vitro em meios sequenciais com hormônios e espermatozóides, Tese (doutorado) - Universidade Estadual Paulista, Faculdade de Ciências Agrárias e Veterinárias, Jaboticabal, 2010. Disponível em:

<http://www.fcav.unesp.br/download/pgtrabs/ra/d/2425.pdf>. Acesso em: 16 mar. 2014.

CHIAN, R.C..; BUCKETT, W. M.; TAN, SEANG-LIN. In vitro maturation of human oocytes.

Reproductive Biomedicine on line, 8: 148-166, 2003.

DAYAN, A.; WATANABE, M. R.; WATANABE, Y. F. Fatores que interferem na produção comercial de embriões FI. Arq Facul Vet UFRGS. V. 28, p. 181-185, 2000.

FERREIRA, E. M.; VIREQUE, A. A.; ADONA, P. R.; MEIRELLES, F. V.; FERRIANI, R. A.; NAVARRO, P. A. A. S. Maturação citoplasmática de oócitos bovinos: aquisição de competência para o 
desenvolvimento, Rev Bras Reprod Anim, Belo Horizonte, v.32, n.3, p.172-181, jul./set. 2008. Disponível em:

<http://www.cbra.org.br/pages/publicacoes/rbra/download/RB166\%20Ferreira\%20vr3\%20pag17 2-181.pdf>. Acesso em: 25 abr. 2014.

GALLI, C.; CROTTI, G.; NOTARI, C. Embryo production by ovum pick up from live donors. Theriogenology, West Lafayette, v. 55, p. 1341-1357, 2000.

http://dx.doi.org/10.1016/S0093-691X(01)00486-1

HEWITT, D. A.; WATSON, P. F.; ENGLAND, G. C. Nuclear staining and culture requirements for in vitro maturation of domestic bitch oocytes. Theriogenology, v.49, p.10831128, 1998. http://dx.doi.org/10.1016/S0093-691X(98)00058-2

NORDBERG, J.; ARNÉR, E. S. J. Reactive oxygen species, antioxidants and the mammalian thioredoxin system. Free Radic Biol Med, v.31, p.1287-1312, 2001.

http://dx.doi.org/10.1016/S0891-5849(01)00724-9

LONERGAN, P.; 1992. Studies in the in vitro maturation, fertilization and cultire of bovine follicular oocytes. PHD Thesis, National University of Ireland, Dublin. 1992.

LONERGAN, P.; RIZOS, D.; GUTIERREZ-ADAN, A.; FAIR, T.; BOLAND, M. P. Oocyte and embryo quality: effect of origin, culture conditions and gene expression patterns. Reprod Domest Anim, County Dublin, v. 38, p. 259-267, 2003.

SAND, C. V. Influência da L-Glutamina exógena nas defesas antioxidantes e na curva de tolerância à glicose, em modelo animal, Dissertação (Mestrado) - Universidade Federal de Santa Catarina, Programa de Pós-Graduação em Farmácia, Florianópolis, SC, 2005.

SILVA, G. M.; ARAÚJO, V. R.; DUARTE A. B. G. et al. Papel dos antioxidantes no cultivo in vitro de células ovarianas. Rev. Bras. Reprod. Anim., v.35, p.315-326, 2011. Disponível em:< http://www.cbra.org.br/pages/publicacoes/rbra/v35n3/pag315-326.pdf >. Acesso em: 14 abr. 2013.

SIRARD, M. A.; RICHARD, F.; BLONDIN, P.; ROBERT, C. Contribution of the oocyte to embryo quality. Theriogenology, Québec, v. 65, p. 126-136, 2006. http://dx.doi.org/10.1016/j.theriogenology.2005.09.020

THOMAS, F. H.; LEASK, R.; SRSEN, V.; RILEY, S. C.; SPEARS, N.; TELFER, E. E. Effect of ascorbic acid on health and morphology of bovine preantral follicles during long-term culture. J Reprod Fertil, v.122, p.487-495, 2001. http://dx.doi.org/10.1530/rep.0.1220487

WANG, X.; FALCONE, T.; ATTARAN, M.; GOLDBERG, J. M.; AGARWAL, A.; SHARMA, R. K. Vitamin C and Vitamin $E$ supplementation reduce oxidative stress-induced embryo toxicity and improve the blastocyst development rate. Fertil Steril, Ohio, v. 78, p. 1272-1277, 2002.

http://dx.doi.org/10.1016/S0015-0282(02)04236-X 